\title{
Factors influencing the incidence and severity of oral mucositis in patients undergoing autologous stem cell transplantation
}

\author{
By Prisco T. Salvador
}

\section{Abstract}

This retrospective descriptive study documented the frequency of oral mucositis and examined the impact of certain variables in the development of oral mucositis in autologous stem cell transplants. Oral mucositis occurred in $90 \%$ of the patients, but $53.57 \%$ developed ulcerations. On average, oral mucositis started on day seven, lasted for six days and resolved on day 13 after the initiation of chemotherapy. Sodium bicarbonate mouthwash and mucositis mouthwash were commonly used interventions; $72.92 \%$ of the interventions were used as secondary prevention. Oral mucositis was significantly associated with diagnosis (lymphoma), chemotherapy (etoposide and melphalan), and level of prevention (secondary). Diagnosis (lymphoma), chemotherapy (etoposide and melphalan), serum creatinine (peak level), and level of prevention (secondary) were independent predictors of oral mucositis.

\section{Introduction}

Oral mucositis remains a common and painful side effect of highdose chemotherapy as a conditioning regimen in autologous stem cell transplantation (ASCT). Patients undergoing ASCT may develop signs and symptoms of oral mucositis within a week after the initiation of chemotherapy. The incidence of oral mucositis in hematopoietic stem cell transplants is estimated at 80\% (National Cancer Institute, 2002).

Several risk factors have been identified as contributing to the severity of oral mucositis in ASCT patients (Berger \& Eilers, 1998; Rapoport et al., 1999). These factors can be classified into patientrelated factors (i.e., type of malignancy, oral hygiene during treatment, age, renal status) and treatment-related factors (i.e., type of chemotherapy, total body irradiation).

Over the last decade, oral mucositis has been the focus of numerous studies and articles that emphasize the importance and benefits of standardized oral hygiene protocols (Larson et al., 1998; Yeager, Webster, Crain, Kasow, \& McGuire, 2000; Stricker \& Sullivan, 2003). Moreover, the implementation of evidence-based oral care guidelines, protocols, and standards may have the potential to minimize the occurrence or reduce the severity of oral mucositis.

In the absence of literature on the optimal prevention and management of oral mucositis, the need for further research to explore the phenomenon of oral mucositis prevention must continue. Hence, this study was conducted to document the frequency of oral mucositis and identify influencing factors in the development of oral mucositis in ASCT patients. The research questions of this study included: (1) What is the incidence of oral mucositis in ASCT patients?, (2) What are the patterns of onset, severity, and resolution of oral mucositis in ASCT patients?, (3) What are the types of intervention and levels of prevention as an intervention used by ASCT patients in preventing the occurrence of oral mucositis?, and (4) Do age, gender, diagnosis, chemotherapy, serum creatinine level, body mass index, and level of prevention as an intervention have an effect in the development of oral mucositis in ASCT patients?

\section{Methods}

A retrospective descriptive design was used to document the frequency of oral mucositis and identify influencing factors in the development of oral mucositis in ASCT patients. Data were collected from 140 charts of ASCT patients who were admitted at a university hospital in southern Ontario, Canada, in the years 2001 and 2002. The criteria for eligibility were: (a) adult over 18 years of age, diagnosed with multiple myeloma (MM), Hodgkin's disease (HD), or nonHodgkin's lymphoma (NHL); (b) use of granulocyte colony stimulating factors (GCSF) as part of the treatment; and (c) type of chemotherapy (melphalan or etoposide and melphalan).

Before data collection started, the researcher obtained an approval letter from the hospital's Research Ethics Board (REB) to conduct a retrospective chart review study for research purposes. With the REB approval on hand, the director of the ASCT program provided the researcher a complete list of patients as required by the study.

The data collection tool used in the study was created by the researcher. The content was reviewed by two medical oncologists and two oncology nurses to ensure consistency, validity, and reliability of the tool. The researcher took approximately 30 minutes to complete the data collection form while reviewing each chart. Patient's confidentiality was maintained throughout the study by assigning each patient a code number that was known only to the researcher. All completed data collection forms were stored in a locked filing cabinet accessible only to the researcher and will remain stored for six years after the study.

There are three components of the data collection tool: demographic information; interventions and levels of prevention as an intervention; and patterns of onset, severity, and resolution of oral mucositis (dependent variable). Patient's demographic information includes age, gender, diagnosis, chemotherapy, serum creatinine levels (baseline/peak), and body mass index (BMI) (independent variables). Interventions comprise oral care agents used to prevent the occurrence or reduce the severity of signs and symptoms of oral mucositis. Levels of prevention as an intervention (independent variable) refer to primary and secondary preventions as mentioned in the Neuman systems model (1995). An intervention is primary prevention when used before signs and symptoms of oral mucositis occur. An intervention is secondary when used with the onset of signs and symptoms of oral mucositis.

To determine the onset, severity, and resolution of oral mucositis in each patient, a timeline was formulated and categorized into three phases of ASCT: pre-transplant, day -4 to -1 ; transplant, day 0; and post-transplant, day +1 to 12 . High-dose chemotherapy (HDC) was administered either on days -4 and -3 for patients diagnosed with HD and NHL or on day -2 for patients diagnosed with MM. On day 0, patients received their own stem cells or bone marrow that were previously collected and cryopreserved. From days +1 to 12 , patients were expected to experience periods of myelosuppression and severe neutropenia before their blood cell counts started to recover into normal range. Development of oral mucositis in ASCT patients onset, severity, and resolution - may occur any time from pretransplant, transplant, or post-transplant phase. The degree of onset, severity, and resolution of oral mucositis in each patient was measured using the World Health Organization (1979) criteria for grading oral mucositis: 0 - none or normal; I - erythema or mild soreness; II painful erythema, edema, or ulcers; III - painful erythema, edema, or ulcers, but cannot eat; and IV - requires parenteral or enteral support.

Descriptive statistics, frequency distribution and measures of central tendency (median and mean) were used to profile the patient's

Prisco T. Salvador, RN, MScN, is a Staff Nurse, Clinical Teaching Unit, Princess Margaret Hospital, Toronto. 
demographic characteristics and other study variables: incidence, onset, severity, and resolution of oral mucositis; and interventions and levels of prevention as an intervention. To examine the impact of each characteristic in the development of oral mucositis, univariate analysis was used. Chi-square test and univariate logistics model were used for categorical and continuous variables respectively. Then, a multiple logistics regression model was fitted to identify independent risk factors. Relationship of predictor variable with oral mucositis was significant if $\mathrm{p}$-value was $<0.05$.

\section{Results}

A total of 140 ASCT patients constituted the study sample. Patients ranged in age between 19 and 72 years with a mean age of 54.96 years. There were more males (84) than females (56) and a preponderance of patients diagnosed with MM (97) and treatment protocol of melphalan (43). Oral mucositis (Grades I-IV) occurred in 126 of the 140 patients, an incidence rate of $90 \%$. The remaining 14 (10\%) patients, 13 patients with MM and 1 patient with HD, did not experience any manifestations of oral mucositis during their treatment. Of the $126(90 \%)$ patients who had oral mucositis, $73.02 \%$ of the patients experienced the onset of signs and symptoms of oral mucositis by day seven after the initiation of HDC. The days of onset ranged between three and 10 with a median of seven and mean of 6.63. Severity of oral mucositis (Grades I - IV) peaked and persisted for six days with a range between two and 10, median of six and mean of 6.26. Moderate to more severe (Grades II - IV) oral mucositis occurred in $53.57 \%(75 / 140)$ of the study sample. Patients showed signs and symptoms of resolution or healing of their oral mucositis between days 10 and 16 after the initiation of HDC. Resolution of oral mucositis in $91.27 \%$ of the patients took place on or before day 14 .

Sodium bicarbonate mouthwash and mucositis mouthwash (a mixture of nystatin and xylocaine viscous) were the most commonly used interventions in both levels of prevention as an interventionprimary and secondary. Primary prevention was used as intervention by $27.08 \%$ of ASCT patients. Secondary prevention was used by $72.92 \%$ of ASCT patients.

Statistically significant relationships with oral mucositis (Table One) were observed in chemotherapy, diagnosis, and level of prevention with $\mathrm{p}$-values of $<=0.05$. Body mass index $(\mathrm{p}=0.0764)$ and serum creatinine with peak level $(\mathrm{p}=0.0743)$ were marginally significant. Age and gender showed no statistical significance. The result of the modelling analysis (Table Two) suggests that creatinine level (peak) $(p=0.0436)$, diagnosis and chemotherapy $(p=0.0042)$,

Table One: Relationship of predictor variables with incidence of oral mucositis

\begin{tabular}{|l|l|c|c|}
\hline Variables & Categories & $\begin{array}{c}\text { \% of oral } \\
\text { mucositis }\end{array}$ & P-value \\
\hline Age & $<50$ years & 47.06 & 0.3815 \\
& $>=50$ & 55.66 & \\
\hline Gender & Male & 41.00 & 0.1665 \\
& Female & 60.71 & \\
\hline Chemotherapy & Melphalan & 43.4 & $0.0003 *$ \\
protocol & Etoposide/Melphalan & 76.74 & \\
\hline Diagnosis & Multiple Myeloma & 43.30 & $0.0003 *$ \\
& Lymphoma (HD/NHL) & 76.74 & \\
\hline Serum \\
creatinine level & Baseline & & 0.5701 \\
\hline Body mass & Peak & & 0.0743 \\
index & \multicolumn{3}{|l}{} \\
\hline Level of & Primary & 41.67 & 0.0764 \\
\hline prevention & Secondary & 62.50 & \\
\hline Note $* p<=0.05$ & & \\
\hline
\end{tabular}

and level of prevention ( $\mathrm{p}=0.0181$ ) were independent risk factors of oral mucositis. Patients with higher peak creatinine level, diagnosed with lymphoma (HD/NHL), treated with etoposide and melphalan, and used secondary prevention as an intervention had a greater chance of developing moderate to more severe oral mucositis.

\section{Discussion}

This retrospective descriptive study of 140 patients treated with HDC plus ASCT has documented the incidence of oral mucositis (Grades I IV) at $90 \% ; 53.57 \%$ of the patients experienced moderate to more severe (grades II - IV) oral mucositis. The onset of oral mucositis occurred on day seven, its severity (Grades II - IV) peaked and persisted for six days, and resolved by day 13 after the initiation of HDC. Early signs and symptoms of oral mucositis included erythema of the soft palate, buccal mucosa, ventral surface of the tongue, and floor of the mouth; these were manifestations of the direct toxic effects of the chemotherapy on the basal cells of the oral mucosa (Woo et al., 1993). Erythema of the oral mucosa is frequently followed by edema and ulcerations. Interestingly, the severity of oral mucositis occurred during the patients' hematological nadir characterized by myelosuppression and severe neutropenia. Resolution of oral mucositis, on the other hand, coincides with the recovery of the neutrophils (Woo et al., 1993).

While the overall incidence of oral mucositis was $90 \%$ in this study, an incidence of $53.57 \%$ represented an ulcerative oral mucositis which is comparatively less than previous studies. Based on evidence from the literature, patients treated with conditioning regimens for bone marrow or peripheral blood stem cell transplantation have higher incidence of oral mucositis. The National Cancer Institute (2002) estimated the frequency of oral mucositis in hematopoietic stem cell transplants at $80 \%$. Woo et al. (1993) reported the prevalence of ulcerative oral mucositis in their study at $76.3 \%$. In a randomized controlled trial comparing the effectiveness of two oral hygiene care in bone marrow transplants, Borowski and colleagues (1994) observed the development of moderate to more severe mucositis at $89.3 \%$.

The resolution of oral mucositis in this study occurred on day 13 after HDC, which was less than the findings of Woo et al. (1993) at approximately day 18 after HDC. The difference may be attributed to the administration of granulocyte colony stimulating factor (GCSF) to all patients included in this study as part of their treatment. Based on previous studies, GCSF can induce neutrophil proliferation and accelerate peripheral blood neutrophil recovery. Furthermore, GCSF has been effective in reducing the incidence and severity of oral mucositis after chemotherapy (Lieschke et al., 1992; Katano,

Table Two: Multiple logistic regression model of factors influencing the incidence and severity of oral mucositis in ASCT patients

\begin{tabular}{|l|c|c|c|c|c|}
\hline Factor & df & Estimate & SE & $\begin{array}{c}\text { Wald/ } \\
\text { Chi-square }\end{array}$ & $\begin{array}{c}\text { P- } \\
\text { value }\end{array}$ \\
\hline Age & 1 & 0.0144 & 0.0204 & 0.5037 & 0.4779 \\
\hline Gender & 1 & 0.5727 & 0.4281 & 1.7898 & 0.1810 \\
\hline $\begin{array}{l}\text { Body } \\
\text { mass index }\end{array}$ & 1 & -0.0662 & 0.0406 & 2.6562 & 0.1031 \\
\hline $\begin{array}{l}\text { Creatinine } \\
\text { (baseline) }\end{array}$ & 1 & -0.0103 & 0.0137 & 0.5633 & 0.4529 \\
\hline $\begin{array}{l}\text { Creatinine } \\
\text { peak) }\end{array}$ & 1 & 0.0283 & 0.0140 & 4.0701 & $0.0436^{*}$ \\
\hline Chemotherapy & 1 & 1.4712 & 0.5141 & 8.1911 & $0.0042^{*}$ \\
\hline Diagnosis & 1 & 1.4712 & 0.5141 & 8.1911 & $0.0042^{*}$ \\
\hline $\begin{array}{l}\text { Prevention } \\
\text { (level) }\end{array}$ & 1 & 0.9356 & 0.3957 & 5.5908 & $0.0181^{*}$ \\
\hline Note: $* \mathrm{p}<=0.05$ & & & & \\
\hline
\end{tabular}


Nakamura, Matsuo, Iyama, \& Hisatsugu, 1995). Unfortunately, the result of this comparison in incidence of oral mucositis is difficult because of heterogeneity of the patient population, sample size differences, and diversity of chemotherapy protocols used across studies.

The relationship between age, gender, diagnosis, chemotherapy, serum creatinine level, body mass index, level of prevention and oral mucositis were examined using univariate and multivariate analyses. This study found that variables such as age, gender, body mass index, and serum creatinine at baseline were not significantly related to the incidence of oral mucositis. Although more than $55 \%$ of the study sample were older than 50 years, this study does not support the trend for increased prevalence and severity of mucositis in older patients as reported by McCarthy et al. (1998). In older patients, the physiological decline in renal function with age may result in increased chemotherapy toxicity including greater vulnerability to oral mucositis (Balducci \& Mowry, 1992). Surprisingly, $40 \%$ of the study sample were females and over $60 \%$ of them developed oral mucositis, but gender was not statistically significant as a risk factor. The finding of this study on gender as a risk factor is in agreement with that of McCarthy et al. (1998).

In a study by Raber-Durlacher et al. (2000), patients with solid tumours and a body mass index $(\mathrm{BMI})<20$ were slightly more susceptible to the development of mucositis than those patients with $\mathrm{BMI}>20$. In contrast, the BMI of the ASCT patients was $>20$ and showed marginal significance (0.0764) in relation to the development of oral mucositis. More often than not, patients with ulcerative oral mucositis may not be able to eat or even drink for a week or longer due to extreme pain associated with swallowing; this condition may result in more severe mucositis through impairment of mucosal regeneration (Sonis, 1993). ASCT patients with ulcerative oral mucositis may benefit from early intervention through meticulous oral assessment and multidisciplinary approach.

\section{References}

Balducci, L., \& Mowry, K. (1992). Pharmacology and organ toxicity of chemotherapy in older patients. Oncology, 6, 62-68.

Beck, S. (1979). Impact of a systematic oral care protocol on stomatitis after chemotherapy. Cancer Nursing, 2, 185-199.

Berger, A., \& Eilers, J. (1998). Factors influencing oral cavity status during high-dose antineoplastic therapy: A second data analysis. Oncology Nursing Forum, 25, 1623-1626.

Borowski, B., Benhamou, E., Pico, J.L., Laplanche, A., Margainaud, J.P., \& Hayat, M. (1994). Prevention of oral mucositis in patients treated with high-dose chemotherapy and bone marrow transplantation: A randomized controlled trial comparing two protocols of dental care. Oral Oncology, European Journal of Cancer, 30B, 93-97.

Dodd, M.J., Dibble, S.L., Miaskowski, C., MacPhail, L., Greenspan, D., Paul, S.M., et al. (2000). Randomized clinical trial of the effectiveness of three commonly used mouthwashes to treat chemotherapy-induced mucositis. Oral Surg Oral Med Oral Pathol Oral Radiol Endod, 90(1), 39-47

Katano, M., Nakamura, M., Matsuo, T., Iyama, A., \& Hisatsugu, T. (1995). Effect of granulocyte colony stimulating factor (G-CSF) on chemotherapy-induced oral mucositis. Surgery Today, 25, 202-206.

Larson, P.J., Miaskowski, C., MacPhail, L., Dodd, M.J., Greenspan, D., Dibble, S.L., et al. (1998). The pro-self mouth aware program: An effective approach for reducing chemotherapy-induced mucositis. Cancer Nursing, 21(4). 263-268.

Lieschke, G., Ramenghi, U., O’Connor, M., Sheridan, W., Szer, J., Morstyn, G. (1992). Studies of oral neutrophil levels in patients receiving G-CSF after autologous marrow transplantation. British Journal of Haematology, 82, 589-95.

McCarthy, G.M., Awde, J.D., Ghandi, H., Vincent, M., \& Kocha, W.I. (1998). Risk factors associated with mucositis in cancer patients receiving 5-fluorouracil. Oral Oncology, 34, 484-490.
This study showed a marginal relationship between serum creatinine with peak level after chemotherapy and oral mucositis; it is also an independent predictor of oral mucositis. This finding suggests that ASCT patients with increased serum creatinine level posttransplant are at greater risk of experiencing severe mucositis. ASCT patients should have their serum creatinine levels monitored daily to detect an increasing level and may benefit from an early intervention.

Diagnosis, chemotherapy, and level of prevention were consistently related to the incidence of oral mucositis and as independent predictor variables of mucositis. These findings indicate that ASCT patients diagnosed with lymphoma (HD/NHL), treated with etoposide and melphalan, and used secondary prevention as an intervention are at greater risk of experiencing severe mucositis. The incidence of oral complications is generally higher in hematological malignancies than in solid tumours due to the degree of immunosuppression experienced by this population (Raber-Durlacher et al., 2000). In light of these findings, patients for ASCT may benefit from the use of primary prevention as an intervention. Several studies documented the benefits of systematic oral assessment and adherence to an oral care protocol as a prophylactic intervention in minimizing or preventing the occurrence of oral mucositis (Beck,1979; Larson et al., 1998; Dodd et al., 2000).

The data collection method used in this study relied primarily on the chart documentation of different health care professionals nurses, physicians, pain specialists, and nutritionists. Several variables were probably under-reported. Moreover, data were not reported for research purposes, therefore omissions or mistakes potentially serious sources of errors - can not be verified.

A prospective descriptive study is currently under consideration to evaluate the effects of such factors as preventive dental care, diagnosis, conditioning regimens, use of comprehensive oral care hygiene, and the use of granulocyte colony stimulating factors in the development of ulcerative oral mucositis. An important component of the study is the use of a valid and reliable oral assessment tool.

National Cancer Institute. (2002). Oral complications of chemotherapy and head/neck radiation (PDQ). Retrieved October 30, 2003, from http://www.cancer.gov/cancerinfo/pdq/supportivecare/oral complications/HealthProfessional

Neuman, B. (1995). The Neuman systems model (3rd ed.). Norwalk, CT: Appleton \& Lange.

Raber-Durlacher, J.E., Weijl, N.I., Abu Saris, M., de Koning, B., Zwinderman, A.H., \& Osanto, S. (2000). Oral mucositis in patients treated with chemotherapy for solid tumours: A retrospective analysis of 150 cases. Supportive Care in Cancer, 8, 366-371.

Rapoport, A.P., Miller Watelet, L.F., Linder, T., Eberly, S., Raubertas, R.F., Lipp, J., et al. (1999). Analysis of factors that correlate with mucositis in recipients of autologous and allogenic stem cell transplants. Journal of Clinical Oncology, 17, 2446-2453.

Sonis, S.T. (1993). Oral complications of cancer therapy. In V.T. De Vita, S. Hellman, \& S.A. Rosenberg (Eds.), Cancer: Principles and practices of oncology (4th ed.) (p. 2385-2394). Philadelphia, PA: Lippincott.

Stricker, C.T., \& Sullivan, J. (2003). Evidence-based oncology oral care clinical practice guidelines: Development, implementation, and evaluation. Clinical Journal of Oncology Nursing, 7(2), 222-227.

Woo, S., Sonis, S.T., Monopoli, M.M., \& Sonis, A.L. (1993). A longitudinal study of oral ulcerative mucositis in bone marrow transplant recipients. Cancer, 72, 1612-1617.

World Health Organization. (1979). Handbook for reporting results of cancer treatment. Geneva, Switzerland: WHO.

Yeager, K.A., Webster, J., Cram, M., Kasow, J., \& McGuire, D.B. (2000). Implementation of an oral care standard for leukemia and transplantation patients. Cancer Nursing, 23, 40-47. 